\title{
A strategy for developing a hammerhead ribozyme for selective RNA cleavage depending on substitutional RNA editing
}

\author{
MASATORA FUKUDA, ${ }^{1}$ KEI KURIHARA, YASUYOSHI TANAKA, and MASANOBU DESHIMARU \\ Department of Chemistry, Faculty of Science, Fukuoka University, Jonan-ku, Fukuoka 814-0180, Japan
}

\begin{abstract}
Substitutional RNA editing plays a crucial role in the regulation of biological processes. Cleavage of target RNA that depends on the specific site of substitutional RNA editing is a useful tool for analyzing and regulating intracellular processes related to RNA editing. Hammerhead ribozymes have been utilized as small catalytic RNAs for cleaving target RNA at a specific site and may be used for RNA-editing-specific RNA cleavage. Here we reveal a design strategy for a hammerhead ribozyme that specifically recognizes adenosine to inosine (A-to-I) and cytosine to uracil (C-to-U) substitutional RNA-editing sites and cleaves target RNA. Because the hammerhead ribozyme cleaves one base upstream of the target-editing site, the base that pairs with the targetediting site was utilized for recognition. RNA-editing-specific ribozymes were designed such that the recognition base paired only with the edited base. These ribozymes showed A-to-I and C-to-U editing-specific cleavage activity against synthetic serotonin receptor $2 \mathrm{C}$ and apolipoprotein $\mathrm{B}$ mRNA fragments in vitro, respectively. Additionally, the ribozyme designed for recognizing A-to-I RNA editing at the $Q / R$ site on filamin $A$ (FLNA) showed editing-specific cleavage activity against physiologically edited FLNA mRNA extracted from cells. We demonstrated that our strategy is effective for cleaving target RNA in an editing-dependent manner. The data in this study provided an experimental basis for the RNA-editing-dependent degradation of specific target RNA in vivo.
\end{abstract}

Keywords: substitutional RNA editing; A-to-I RNA editing; C-to-U RNA editing; hammerhead ribozyme

\section{INTRODUCTION}

RNA editing, a mechanism that alters genomic information after transcription, is exhibited in various forms, including base insertions, deletions, and substitutions (Maas and Rich 2000; Bass 2002; Farajollahi and Maas 2010; Nishikura 2010). In particular, base-modification editing of adenosine (A) to inosine (I) within pre-mRNA was shown to be a widespread form of information recording of mRNA codons in higher eukaryotes (Nishikura 2010; Pullirsch and Jantsch 2010). A-to-I substitutions in protein-coding sequences may result in codon changes because inosine is decoded as guanosine by the ribosome during translation. The most studied A-to-I editing events in coding RNAs are those involving the glutamate receptor subunit B (GRIA2) (Sommer et al. 1991) and the serotonin $2 \mathrm{C}$ receptor (HTR2C) pre-mRNAs (Burns

\footnotetext{
${ }^{1}$ Corresponding author

E-mail masatora@fukuoka-u.ac.jp

Article published online ahead of print. Article and publication date are at http://www.rnajournal.org/cgi/doi/10.1261/rna.033399.112.
}

et al. 1997). For GRIA2, RNA editing regulates the desensitization kinetics of the receptor and the $\mathrm{Ca}^{2+}$ permeability of the ion channel (Higuchi et al. 1993; Seeburg and Hartner 2003). HTR2C pre-mRNA is edited at five adenosines, resulting in a change in three consecutive amino acids (Werry et al. 2008). This editing leads to 24 different isoforms and modulates receptor $\mathrm{G}$ protein interaction and trafficking (Werry et al. 2008). In contrast, cytosine to uracil (C-to-U) RNA editing, which is another type of substitutional RNA editing, changes cytosine to uridine through deamination (Blanc and Davidson 2003). The nuclear transcript encoding intestinal apolipoprotein $\mathrm{B}(A P O B)$ is subject to C-to-U RNA editing; $A P O B$ mRNA editing changes a CAA codon to a UAA stop codon, resulting in the generation of a truncated protein (Wang et al. 2003). As described above, A-to-I and C-to-U RNA editing is related to an important mechanism for regulating genetic plasticity by generating alternative protein products. Since substitutional RNA editing in coding regions plays a crucial role in the control of biological processes, abnormal editing often causes serious diseases (Maas et al. 2006). Recently, genetic screening studies have identified 
additional RNA-editing targets (Levanon et al. 2005; Gommans et al. 2008; Li et al. 2009), particularly in proteincoding sequences of receptors, ion channels, and other proteins (Li et al. 2009; Pullirsch and Jantsch 2010). Although high-throughput sequencing data suggests that substitutional RNA editing regulates protein function by altering the amino acid sequence, the detailed biological functions of this editing are unclear.

The ability to control site-specific substitutional RNA editing makes it an attractive tool for analyzing and regulating biological processes related to RNA editing. We utilized the ribozyme for selective digestion of target RNA at particular sites that are subjected to substitutional RNA editing. The hammerhead ribozyme (HHR), which is the smallest catalytic RNA motif, can cleave the RNA phosphodiester bond at a specific site. Minimal trans-cleaving HHRs have been engineered by modifying natural HHRs (Uhlenbeck 1987; Haseloff and Gerlach 1988) and have been utilized for RNA-mediated gene regulation to inhibit target gene expression in vivo (Goodchild 2002; Citti and Rainaldi 2005; Tedeschi et al. 2009). HHR consists of two sequence elements, including a conserved central catalytic core and two hybridizing arms for recognizing the target sequence. A target-specific HHR has been created by altering the sequences of the hybridizing arms against the target RNA sequence according to simple Watson-Crick basepairing rules. Many different catalytic core sequences are available for target RNAs. Designed HHRs can bind to and cleave target RNA by using sequences that are complementary to the hybridizing sequences flanking an $\mathrm{NHH}$ triplet at the phosphodiester bond $3^{\prime}$ to the second $\mathrm{H}$, where $\mathrm{N}$ is any nucleotide and $\mathrm{H}$ can be either $\mathrm{A}, \mathrm{C}$, or $\mathrm{U}$ (Kore et al. 1998). Additionally, mutation-specific mRNA cleavage also has been achieved using a designed HHR in vitro and in vivo (Scherr et al. 1997; Lewin et al. 1998; Dawson and Marini 2000). These mutation-specific properties of HHRs were generated on the basis of the cleavage preference for the $\mathrm{NHH}$ triplet. Although several HHRs are available for mutation-specific cleavage of target RNA, no trans-acting HHRs that specifically recognize substitutional RNA editing have been reported.

Here we report the construction of artificial ribozymes that specifically cleave target RNA only when the site of interest has been subjected to substitutional RNA editing. In this study, NHH specificity was not used; instead, an HHR with RNA-editing-specific cleavage activity was designed by substituting the hybridizing arm nucleotide that was closest to the catalytic core sequence. The base of this nucleotide can be utilized as an editing recognition base because HHR cleavage activity is highly affected by base-pairing at this position (Zoumadakis et al. 1994; Werner and Uhlenbeck 1995). The initial editing-specific HHR, parental HHR, was designed to cleave $5^{\prime}$ to the target-editing site. This HHR design provides Watson-Crick base-pairing between the recognition base and the target-editing site (Fig. 1). On the

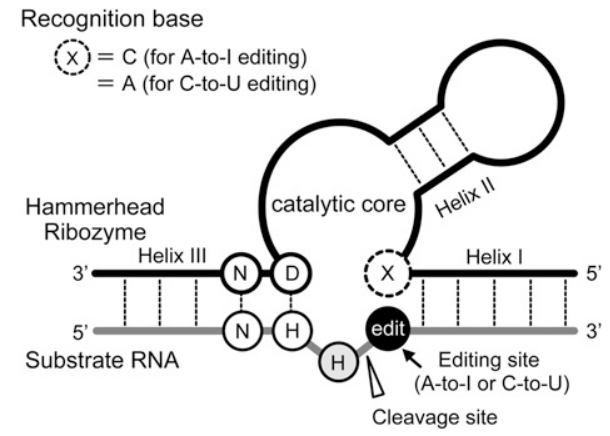

FIGURE 1. Schematic representation of hammerhead ribozyme (HHR) for specific cleavage of substrate RNA subjected to substitutional RNA editing. Ribozyme and substrate RNA are shown as black and gray lines, respectively. Dashed lines indicate the base-pair interactions. Ribozyme contains three helices: Helix I and Helix III are in the hybridizing arms for recognizing the target sequence, and Helix II is in the catalytic core. On the substrate RNA, the cleavage site and editing site are denoted by gray and black circles, respectively. To introduce the ability for editing-specific cleavage into the HHR, the recognition base (shown as a circle with dotted outline) is designed to pair only with the edited base at the editing site. In this study, the recognition base $\mathrm{C}$ was used for A-to-I editing, and A was used for $\mathrm{C}$-to- $\mathrm{U}$ editing. Position of the $5^{\prime}-\mathrm{NHH}-3^{\prime}$ triplet for $\mathrm{HHR}$ specificity is shown in this figure, where $\mathrm{N}$ is any nucleotide, and $\mathrm{H}$ is either $\mathrm{A}$, C, or U. D is any nucleotide except C.

basis of this HHR, the recognition base was altered to pair only with the edited base, not with the unedited base. For A-to-I RNA-editing-specific cleavage, cytosine was used as a recognition base. Similarly, guanosine was changed to adenosine for C-to-U-specific cleavage. These designed ribozymes showed higher cleavage activity against both A-to-I edited HTR2C RNA fragments and C-to-U edited $A P O B$ RNA fragments than against the nonedited RNAs in vitro. We also demonstrated that the ribozyme designed for A-to-I RNA-editing recognition at the Q/R site on filamin A (FLNA) showed editing-specific cleavage activity against physiologically edited FLNA mRNA extracted from cells.

\section{RESULTS}

\section{Ribozyme design for RNA-editing-specific cleavage based on HHR structure}

Because of their specificity for the NHH base triplet, transacting HHRs have been used as mutation-specific RNA cleavage tools (Goodchild 2002; Citti and Rainaldi 2005; Tedeschi et al. 2009). However, HHR cleavage activity is significantly affected by not only the $\mathrm{NHH}$ preference, but also by base-pairing close to the catalytic core sequence (Werner and Uhlenbeck 1995). By utilizing this HHR base as an editing-recognition base, a substitutional RNA-editing-specific ribozyme can be designed on the basis of the base-pairing ability of the HHR base (Fig. 1). To assess the effectiveness of our designed ribozyme for editing-specific cleavage, we selected an A-to-I editing site on HTR2C mRNA, 
the $\mathrm{C}$ site, as a target-editing site. To construct a ribozyme with A-to-I editing-specific cleavage, parental HHR (HR-HTR2C) was designed to contain the catalytic core sequence and 16 bases complementary to HTR2C RNA for cleavage of the site $5^{\prime}$ to the $\mathrm{C}$ according to a common method (Fig. 2A). The catalytic core sequence from $\mathrm{HH} 16$ was used in this study (5'-CUGAUGAGGCCGAAAGGCCGAA-3'), which is composed of a consensus sequence (5'-CUGANGA-GAA-3') and a stem-loop structure containing a 4-bp GCGC duplex and a GAAA tetraloop (Scott et al. 1995). Since the sequence context of the target cleavage site in this design, a GUA triplet, was selected on the basis of the $\mathrm{NHH}$ rule, parental HHR was expected to exhibit cleavage activity against HTR2C RNA. In the parental HHR construct, the recognition base forms a $\mathrm{U}-\mathrm{A}$ ( $\mathrm{A}$ is the editing site) base pair with the $\mathrm{C}$ site. In the second step, an editing-specific ribozyme was designed by changing the $\mathrm{U}$ at the recognition base to $\mathrm{C}$ so as to form a base pair only when the A of the $\mathrm{C}$ site was substituted with I by A-to-I RNA editing (Fig. 2A). Inosine forms a wobble base pair with cytosine that is identical to a $\mathrm{G}-\mathrm{C}$ base pair, except that the $\mathrm{I}-\mathrm{C}$ base pair lacks the hydrogen-bond donor ability that is present at the 2-amino group of $\mathrm{G}$. As a result, the ribozyme

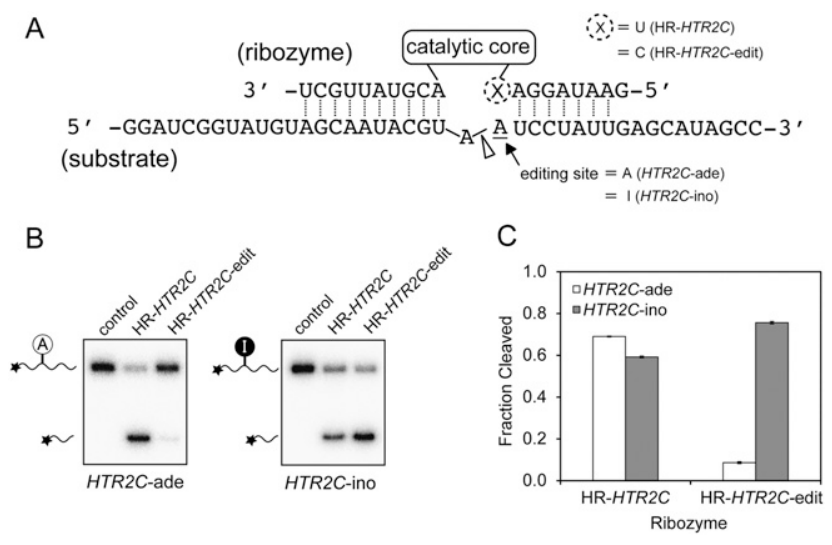

FIGURE 2. A-to-I editing-specific cleavage of ribozyme against HTR2C mRNA fragment. (A) Sequences of ribozymes and substrates of synthetic HTR2C mRNA fragment. Parental ribozyme HR-HTR2C was designed to cleave $5^{\prime}$ to the editing site. HR-HTR2C-edit was generated from HR-HTR2C by altering the recognition base to $\mathrm{C}$ for A-to-I editing recognition. The recognition base of the ribozyme is marked by a circle with a dotted outline. Sequence of a catalytic core for all ribozyme constructs is $5^{\prime}$-CUGAUGAGGCCGAAAGGCCGAA-3'. The cleavage site is indicated by a white arrowhead, and target A-to-I editing site of $\mathrm{A}$ is underlined in the substrate sequence. Unedited and edited substrates are denoted as HTR2C-ade and HTR2C-ino, respectively. $(B)$ Analysis of A-to-I-editing-specific cleavage by ribozyme using denaturing PAGE $(15 \%)$. The cleavage reaction was performed with HTR2C-ade (left) and HTR2C-ino (right) in the presence of an excess of ribozyme at $37^{\circ} \mathrm{C}$ for $1 \mathrm{~h}$. Positions of the $5^{\prime}$-labeled substrates $(\mathrm{S})$ and of the resulting $5^{\prime}$-cleavage products $(\mathrm{P})$ are indicated. $(C)$ Summary of the cleavage ratios obtained from experiment $B$ for HR-HTR2C and HR-HTR2C-edit against HTR2Cade and HTR2C-ino. Intensities of the substrate band (S) and cleavage product band $(\mathrm{P})$ were quantified, and the cleavage ratios were calculated using the expression $\mathrm{P} /(\mathrm{S}+\mathrm{P})$. gains the ability to discriminate the target RNA-editing site through base-pairing recognition. Therefore, the cleavage activity of this ribozyme (HR-HTR2C-edit) was expected to be higher against edited-HTR2C than unedited-HTR2C.

\section{In vitro trans-cleavage activity and specificity of the A-to-I RNA-editing-specific ribozyme against HTR2C RNA fragment}

To assess the cleavage activity and editing specificity of HRHTR2C-edit, in vitro cleavage assays were performed using a synthetic HTR2C RNA fragment containing a C site. In this assay, we used two types of ${ }^{32} \mathrm{P}$-labeled $37-n$ t HTR2C mRNA fragments, one with an $\mathrm{A}$ at the $\mathrm{C}$ site as the unedited substrate (HTR2C-ade) and another with an I as the edited substrate (HTR2C-ino) (Fig. 2A). Substrate RNAs were annealed in the presence of an excess of HR-HTR2C and HRHTR2C-edit ribozymes that had been transcribed in vitro and gel-purified. Cleavage reactions were initiated by the addition of $20 \mathrm{mM} \mathrm{MgCl}_{2}$ at $37^{\circ} \mathrm{C}$. After $1 \mathrm{~h}$, cleavage bands were analyzed using gel electrophoresis, and cleavage ratios were calculated (Fig. 2B,C). In the parental HHR construct (HR-HTR2C), the recognition base forms a $\mathrm{U}-\mathrm{A}$ (A is the editing site) base pair with the $\mathrm{C}$ site. $\mathrm{U}$ can form a base pair with both A and I; hence, the recognition base of HR-HTR2C cannot discriminate between the nonedited and edited $\mathrm{C}$ sites. As a result, HR-HTR2C exhibits cleavage activity against HTR2C RNA in an editing-independent manner. In the case of HR-HTR2C, similar cleavage ratios were observed against HTR2C-ade and HTR2C-ino (0.69 and 0.59 , respectively). In contrast, cleavage ratios using HRHTR2C-edit were much higher against HTR2C-ino than against HTR2C-ade (0.76 and 0.09 , respectively). These results demonstrate that altering the second base of HRHTR2C to form HR-HTR2C-edit confers the A-to-I editingspecific cleavage activity. To analyze the cleavage kinetics for the ribozymes, we next determined trans-cleaving rate constants under single-turnover conditions by using the same experimental method described above (Fig. 3). Figure 3A shows the time course of the cleavage products resulting from the reactions of HR-HTR2C-edit against HTR2C-ade (Fig. 3A, top) and HTR2C-ino (Fig. 3A, bottom). Kinetic analysis results of HR-HTR2C-edit showed a high $k_{\text {cat }}$ value $(0.67 \pm$ $\left.0.011 \mathrm{~min}^{-1}\right)$ with $H$ TR2C-ino and a low $k_{\text {cat }}$ value $(0.01 \pm$ $<0.001 \mathrm{~min}^{-1}$ ) with HTR2C-ade (Fig. 3B; Table 1). In contrast, HR-HTR2C catalyzed the cleavage of both edited and unedited substrates with $k_{\text {cat }}$ values of $0.05 \pm 0.001$ $\mathrm{min}^{-1}$ and $0.44 \pm 0.022 \mathrm{~min}^{-1}$, respectively. The difference in $k_{\text {cat }}$ values for this ribozyme was smaller than that for HRHTR2C-edit. The fraction of product at the end point of the reaction $\left(F_{\infty}\right)$ was observed to be similar when the combinations of the recognition base and editing site were $\mathrm{U}-\mathrm{A}(0.74)$, U-I (0.63), and C-I (0.85), but C-A showed a significantly lower value (0.23). Consistent with the results of previous studies (Werner and Uhlenbeck 1995), we found that the 
A

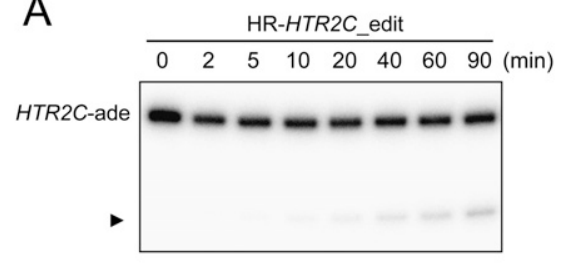

HR-HTR2C_edit

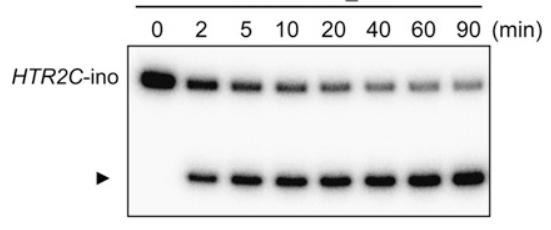

B

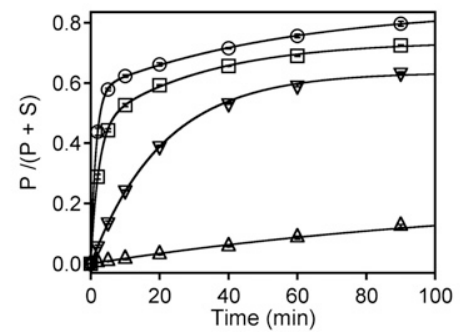

FIGURE 3. Time course of cleavage reaction and kinetic analysis for HR-HTR2C and HR-HTR2C-edit against unedited- and edited-HTR2C RNA fragments under single-turnover conditions. (A) Denaturing PAGE $(15 \%)$ showing the time course of cleavage of HR-HTR2C-edit against $H T R 2 C$-ade (top) and HTR2C-ino (bottom). Positions of the resulting $5^{\prime}$-cleavage products $(\mathrm{P})$ are indicated by arrowheads. $(B)$ Diagrams representing the cleavage ratio as a function of reaction time. Each data for the set of ribozyme and substrate (ribozyme/substrate) are represented as squares (HR-HTR2C/HTR2C-ade), inverted triangles (HR-HTR2C/HTR2C-ino), triangles (HR-HTR2C-edit/HTR2C-ade), and circles (HR-HTR2C-edit/HTR2C-ino). The cleavage ratio at different time points was determined using quantitative scanning of corresponding gel bands and calculated using the expression $\mathrm{P} /(\mathrm{S}+\mathrm{P})$. All data were obtained from two independent experiments. Fitting to a single-exponential equation generated progress curves for the cleavage reactions of HR-HTR2C/HTR2C-ino and HR-HTR2C-edit/HTR2C-ade, and the data obtained for HR-HTR2C/HTR2C-ade and HR-HTR2Cedit/HTR2C-ino were fitted to a double-exponential equation. Kinetic parameters obtained in this experiment are summarized in Table 1.

cleavage activity of HR-HTR2C-edit was highly affected depending on whether a base pair was formed with the recognition base. For HR-HTR2C, the difference in cleavage activity between $\mathrm{U}-\mathrm{A}$ and $\mathrm{U}-\mathrm{I}$ appears to be ordered according to the thermostability of the base pair; a U-I base pair is less stable than a U-A base pair (Serra et al. 2004). These results indicate that editing-specific cleavage activity of the ribozyme can be designed by combining the recognition base and the base of the target-editing site.

\section{C-to-U-editing-specific cleavage for $A P O B$ RNA with the ribozyme}

To determine whether our ribozyme design could be applied for specific cleavage of other types of substitutional RNA editing, we next constructed a C-to-U-editing-specific ribozyme by altering the recognition base. The C-to$\mathrm{U}$-editing-specific ribozyme was designed as a target for $A P O B$ mRNA because it has been shown that C-to-U editing of $\mathrm{C}$ at position 6666 in $A P O B$ mRNA converts a glutamine codon (CAA) to an in-frame stop codon (UAA) (Chen et al. 1987; Powell et al. 1987; Chester et al. 2000). Using a method similar to the method used for A-to-I editing, parental HHR was designed to cleave $5^{\prime}$ to the $\mathrm{C}$-to- $\mathrm{U}$ editing site on $A P O B$ mRNA by using the same catalytic core sequence (Fig. 4A). The C-to-U editing-specific ribozyme (HR-APOB-edit) was generated by introducing $\mathrm{A}$ into the recognition nucleotide such that it could base pair with $\mathrm{U}$ resulting from the C-to-U editing of $A P O B$ RNA. Cleavage activity of HR-APOBedit against the unedited (APOB-cyt) and edited $(A P O B$ uri) substrates was analyzed using an in vitro cleavage assay (Fig. 4B). As shown in Figure 4B, cleavage of $A P O B$ uri was much greater than that of $A P O B$-cyt after a $1-h$ cleavage reaction in the presence of excess ribozyme. Kinetic analysis for HR-APOB-edit (Fig. 4C) showed that the cleavage rates of HR-APOB-edit against $A P O B$-cyt and $A P O B$-uri were significantly different $(0.01 \pm 0.006$ $\min ^{-1}$ and $0.17 \pm 0.008 \mathrm{~min}^{-1}$, respectively). Moreover, a distinct difference was observed between $A P O B$-cyt and $A P O B$-uri in the fraction of product at end point. These data clearly demonstrate the C-to-U-editing-specific cleavage activity of HR-APOB-edit. These results indicated that selecting a combination of a recognition base and target-editing site could be applied to the construction of ribozymes not only for A-to-I-editing-specific cleavage but also for C-to-U-editing-specific cleavage.

\section{A-to-I-editing-specific cleavage for FLNA mRNA extracted from cells}

We next evaluated the efficiency of A-to-I-editing-specific cleavage against a physiological mRNA extracted from cells. FLNA mRNA, which contains the conserved A-to-I RNAediting site $(\mathrm{Q} / \mathrm{R}$ site), was used as the target mRNA because endogenous FLNA mRNA edited following overexpression of adenosine deaminase acts on RNA 2 (ADAR2) in the cell (Nishimoto et al. 2008; Riedmann et al. 2008). The ribozyme for editing-specific cleavage of FLNA mRNA (HR-FLNAedit) was designed using the method described above, and cleavage activity and editing specificity of HR-FLNA-edit were analyzed by an in vitro cleavage assay using synthetic FLNA RNA fragments (Supplemental Fig. S2). The cleavage rate constant and fraction of product at the end point of HR-FLNA-edit against the edited-FLNA RNA fragment were much greater than those of the unedited-FLNA RNA fragment (Table 1; Supplemental Fig. S2); these results were similar to those observed for HR-HTR2C-edit.

In order to assess the efficiency of editing-specific cleavage for HR-FLNA-edit against physiological FLNA mRNA, we 
TABLE 1. Single turnover-cleavage rates of hammerhead ribozymes against edited and nonedited RNA substrates

\begin{tabular}{llcccc}
\hline Ribozyme & Substrate & $k_{\mathrm{a}}\left(\mathrm{min}^{-1}\right)^{\mathrm{a}}$ & $k_{\mathrm{b}}\left(\mathrm{min}^{-1}\right)^{\mathrm{b}}$ & $F_{\infty}{ }^{\mathrm{c}}$ & $F_{\mathrm{a}}{ }^{\mathrm{d}}$ \\
\hline HT-HTR2C & HTR2C-ade & $0.44 \pm 0.022$ & $0.031 \pm 0.003$ & 0.74 & 0.46 \\
& HTR2C-ino & $0.05 \pm 0.001$ & & 0.63 & \\
HT-HTR2C_edit & HTR2C-ade & $0.01 \pm<0.001$ & & 0.23 & \\
& HTR2C-ino & $0.67 \pm 0.011$ & $0.018 \pm 0.001$ & 0.85 & 0.57 \\
HR-APOB-edit & APOB-cyt & $0.01 \pm 0.006$ & & 0.02 & \\
& APOB-uri & $0.17 \pm 0.008$ & & 0.86 & \\
HR-FLNA-edit & FLNA-ade & $0.02 \pm 0.008$ & & 0.03 & \\
& FLNA-ino & $0.12 \pm<0.001$ & & 0.79 & \\
\hline
\end{tabular}

${ }^{a}$ Cleavage rate constant for the rapid process (a).

${ }^{\mathrm{b}}$ Cleavage rate constant for the slow process (b).

${ }^{\mathrm{c}}$ Fraction of product at the end point of the reaction.

${ }^{\mathrm{d}}$ Fraction of product at the end point of the rapid process (a).

2011); however, we evaluated A/G mixed peak heights in order to determine how the reduction of the editing ratio relates to the reaction time in the presence of HR-FLNA-edit (Supplemental Fig. S3). These results showed that HR-FLNAedit cleaved cell-extracted FLNA mRNA in an editing-specific manner.

\section{DISCUSSION}

Substitutional RNA editing serves an important role in biological processes, and the functions of some cellular proteins, such as $H T R 2 C$ and $A P O B$, are regulated by the editing ratio at particular sites on their mRNAs. Since a

prepared edited-FLNA mRNA from HEK293 cells overexpressing ADAR2. We previously established a Tet-ADAR2 cell, which can be stably transfected with the ADAR2expression vector containing the doxycycline (Dox)-inducible expression system. Total RNAs containing a mixture of edited-FLNA mRNA and unedited-FLNA mRNA were obtained from Tet-ADAR2 cells cultured with $5 \mu \mathrm{g} / \mathrm{mL}$ of Dox. If the ribozyme specifically cleaves the edited-FLNA mRNA in the extracted total RNA, the ratio of unedited- to edited-FLNA mRNA should be reduced. Therefore, the efficiency of the editing-specific cleavage can be evaluated by measuring the difference between samples with and without ribozyme in the editing ratio at the $\mathrm{Q} / \mathrm{R}$ site. Furthermore, to assess the possibility of the effect of HHR on FLNA mRNA stability, we synthesized a catalytically inactive HHR based on the HR-FLNA-edit sequence.

The experimental scheme for quantifying the editing ratio is shown in Figure 5A. First, 250 ng of extracted RNA was annealed with an excess of either HR-FLNA-edit or HRFLNA-mut ( $2 \mu \mathrm{M}$ final concentration), followed by cleavage reaction in a buffer containing $20 \mathrm{mM} \mathrm{MgCl}_{2}$. After performing the cleavage reactions for different durations $(1,3,6$, and $24 \mathrm{~h})$, RNA samples were subjected to reverse transcriptase-polymerase chain reaction (RT-PCR) with FLNA-specific primers, followed by direct sequencing of PCR fragments using the fluorescent dideoxy sequencing method (Fig. 5A). To quantify the A-to-I-editing ratio at the $\mathrm{Q} / \mathrm{R}$ site of each sample, $\mathrm{T}$ and $\mathrm{C}$ peak heights were measured in chromatograms generated for sequences amplified using the reverse primer (Fig. 5B). In the reactions with HRFLNA-mut and without HHR, no significant changes of the editing ratio were observed at any time point. In contrast, the editing ratio decreased according to the duration of the HR-FLNA-edit reaction, with ratios of $0.56,0.52,0.47$, and 0.36 at $1,3,6$, and $24 \mathrm{~h}$, respectively. These values were lower than those for the reactions with HR-FLNA-mut and without ribozyme. $\mathrm{A} / \mathrm{G}$ mixed peaks have more variable heights than T/C mixed peaks (Nurpeisov et al. 2003; Eggington et al. method for controlling the editing ratio at a specific site will be useful for analyzing and regulating intracellular processes related to RNA editing, we aimed to reduce the level of mRNA editing by taking advantage of the editing-specific cleavage activity of designed ribozymes. The design used for constructing editing-specific ribozymes in this study involved

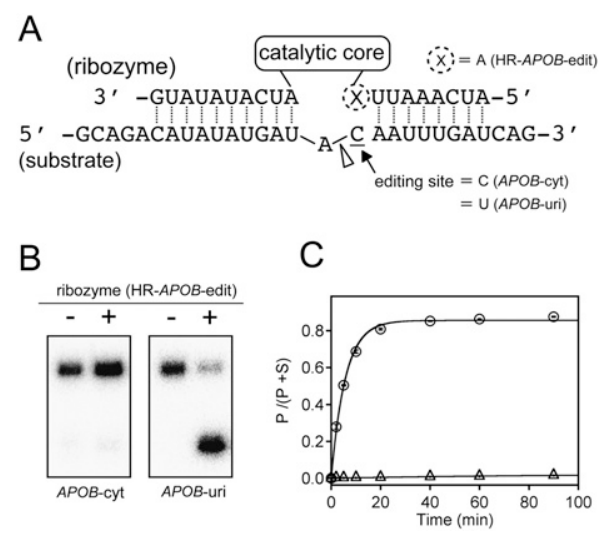

FIGURE 4. C-to-U editing-specific cleavage of ribozyme against the $A P O B$ mRNA fragment. ( $A$ ) Sequences of ribozymes and substrates of the synthetic $A P O B$ mRNA fragment. HR-APOB-edit was generated by introducing $A$ as the recognition base for C-to- $U$ editing recognition. The recognition base of the ribozyme is marked by a circle with a dotted outline, and the sequence of the catalytic core is the same as that of the HR-HTR2C. The cleavage site is indicated by a white arrowhead, and the target C-to- $\mathrm{U}$ editing site of $\mathrm{C}$ is underlined in the substrate sequence. Unedited and edited substrates are denoted as $A P O B$-cyt and $A P O B$-uri, respectively. (B) Analysis of editing-specific cleavage of HR- $A P O B$-edit using denaturing PAGE $(15 \%)$. The cleavage reaction was performed with $A P O B$-cyt (left) and $A P O B$-uri (right) in the presence of excess ribozyme at $37^{\circ} \mathrm{C}$ for $1 \mathrm{~h}$. (C) Time course of cleavage reaction and kinetic analysis for $\mathrm{HR}-A P O B$-edit against unedited and edited $A P O B$ RNA fragments under single-turnover conditions. The diagram represents the cleavage ratio as a function of reaction time and the sets of ribozyme/substrate are shown as triangles (HR-APOB-edit/ $A P O B$-cyt) and circles (HR- $A P O B$-edit/APOB-uri). Data were obtained from two independent experiments. Fitting curves were generated using a single-exponential equation. Kinetic parameters obtained from this experiment are summarized in Table 1. 
A

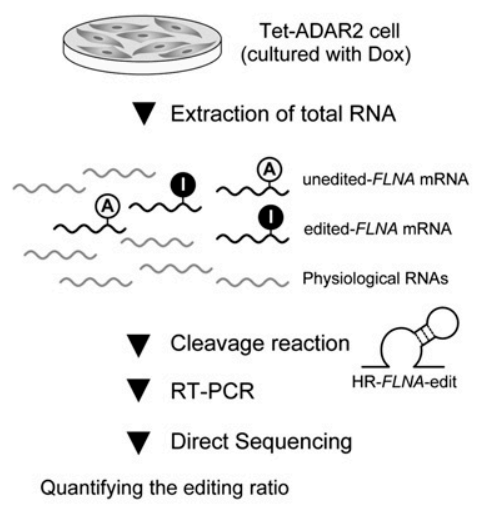

B

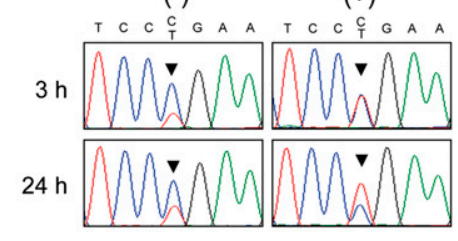

C

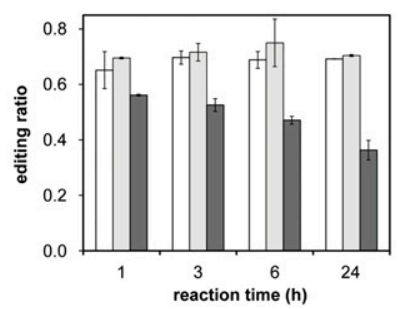

FIGURE 5. In vitro A-to-I editing-specific cleavage of ribozyme against FLNA mRNA extracted from cells. (A) Experimental procedure for analyzing editing-specific cleavage of HR-FLNA-edit against extracted FLNA mRNA by measuring the editing ratio at the Q/R site. Total RNAs containing FLNA mRNA were extracted from Tet-ADAR2 cells and then reacted with HR-FLNAedit to measure editing-specific cleavage. The editing ratio was calculated from the sequence chromatogram generated by direct sequencing of the RT-PCR product from the ribozyme-reacted total RNA. (B) Sequence chromatograms of products with the cleavage reaction of HR-FLNA-edit and HR-FLNA-mut, or in the absence of HHR. Chromatograms, which were generated using reverse FLNA-specific primers, are shown for the sequences obtained from the reaction with HRFLNA-edit for $3 \mathrm{~h}$ (top, right) and $24 \mathrm{~h}$ (bottom, right), and from the incubation without ribozyme for $3 \mathrm{~h} \mathrm{(top,} \mathrm{left)}$ and $24 \mathrm{~h}$ (bottom, left) that were used as controls. (C) Quantification of the editing ratio of FLNA mRNA after the ribozyme reaction. The bar graph shows the editing ratios at the $\mathrm{Q} / \mathrm{R}$ site from the HR-FLNA-edit reaction (dark gray bars), HR-FLNA-mut (light gray bars), and without reaction (white bars). Editing ratios were quantified by measuring the peak heights of $\mathrm{T}$ and $\mathrm{C}$ and calculated on the basis of the peak height of $\mathrm{C}$ divided by $\mathrm{C}+\mathrm{T}$. Results are presented as an average editing ratio and standard deviation from two independent experiments.

altering the recognition base such that it paired only with the edited base, and the designed ribozyme was shown to be very effective for RNA-editing-specific cleavage. Ribozymes designed for A-to-I-editing-specific cleavage showed $>10$-fold higher catalytic rate against edited-HTR2C and edited-FLNA RNA fragments than against unedited RNAs. Similarly, a ribozyme for C-to- $\mathrm{U}$ editing of $A P O B$ RNA had a much higher catalytic rate against the edited- $A P O B$ RNA fragment. These results from our in vitro cleavage assay demonstrated the selective-cleavage activity against an edited substrate. By comparing kinetic analysis data from editing-specific ribozymes against edited substrates, the cleavage rate of HRHTR2C-edit was found to be much higher than that of HRFLNA-edit and HR-APOB-edit, whereas the fractions of product at the end of the reactions were similar (Table 1). These differences in rate may be due to the sequence of the hybridizing arms and their base pairs (Perriman et al. 1992; Zoumadakis and Tabler 1995). The data for HTR2C-ade/ HR-HTR2C and HTR2C-ino/HR-HTR2C-edit (Fig. 2C) were fitted by the double-exponential equation, because single-exponential equations were ineffective for this purpose. While the kinetic analysis was performed using a single turnover reaction with an excess amount of ribozyme against substrate RNA, it seems that two kinds of cleavage reaction mechanisms (fast and slow processes) exist during HTR2C RNA cleavage. The details of the cleavage mechanism could not be evaluated in our experiment; however, comparison of the FLNA and $A P O B$ RNA cleavage suggested that the nature of cleavage is dependent on the sequence context of the substrate RNA. Although the kinetic data could not be compared directly between the data fitted in two different ways, HTR2C-ade/HR-HTR2C and HTR2C-ino/HR-HTR2C-edit were much more active than $H T R 2 C$-ino/HRHTR2C and HTR2C-ade/HR-HTR2Cedit, as reflected by the rate constant of the rapid process and fraction of product at the end-point of the reaction.

As shown in mutation analyses for the HHR at the position corresponding to the recognition base (Zoumadakis et al. 1994; Werner and Uhlenbeck 1995), target-cleavage activity of the designed ribozyme can be controlled on the basis of whether the recognition base can pair with the edited base. Additionally, data for HR-HTR2C with U-A and U-I base pairs also indicates that cleavage activity is affected by the thermostability of the base pair at the recognition base. These results suggest that our design can be applied not only to substitutional RNA editing but also to specific cleavage of other types of base substitutions, including point mutations, by selecting the appropriate combination of recognition base and target base.

HHRs have intrinsic cleavage specificity for $\mathrm{NHH}$ triplets, and this has been exploited to recognize point mutations (Scherr et al. 1997; Lewin et al. 1998; Dawson and Marini 2000). The cleavage activity of trans-HHR is significantly lower when the cleavage site of target RNA is $\mathrm{G}$ (NHH: cleavage site is underlined); therefore, $\mathrm{NHH}$ specificity can be utilized to discriminate between $\mathrm{G}$ and other natural nucleotides (Scherr et al. 1997; Lewin et al. 1998). However, with regard to the recognition of A-to-I RNA editing, this strategy is not available for editing-specific cleavage because target RNAs with inosine at the cleavage site are good substrates for HHR (Koizumi and Ohtsuka 1991). Another strategy of the NHH rule for mutationspecific cleavage is to use the first $\mathrm{H}$ position ( $\underline{\mathrm{HH}}$ : first position of $\mathrm{H}$ is underlined). This nucleotide should form a base pair during HHR cleavage activity, and the most preferable substrate for natural HHR is the NUH triplet (Kore et al. 1998). This position has been used to perform allele-specific cleavage on target RNAs, including the specific site of $U$ and $G$ on type I collagen mRNA (Dawson and 
Marini 2000). Although these discrimination strategies are very effective, they are restricted to a subset of target mutations. This is because the cleavage activity of minimal HHRs against NAH substrates is much lower than that observed with NUH substrates (Kore et al. 1998). On the other hand, $\mathrm{NCH}$ substrates can be efficiently cleaved by replacing a base-pairing nucleotide in the HHR with inosine, and this ribozyme is useful for mutation-specific cleavage (Ludwig et al. 1998). This type of HHR requires chemical modification; therefore, it is not applicable for editing-dependent cleavage in vivo. On the contrary, our strategy was not constrained by the NHH rule; hence, target-cleavage activity of the designed ribozyme can be controlled on the basis of whether the recognition base can pair with the edited base. Additionally, cleavage activity of parental HHR in which the recognition base has not been substituted can be easily designed according to the $\mathrm{NHH}$ rule, and editing-specific HHRs can be obtained by substituting the recognition base without a large alteration of cleavage activity of parental HHR. In this work HHRs recognizing A-to-I and C-to-U substitutional RNA editing were successfully constructed using a baserecognition strategy. By designing novel combinations of base-pairing HHRs, our strategy would be applicable for recognizing almost all combinations of single point mutations.

Altering the recognition base is useful for designing editingand mutation-specific cleavage, even though HHR cleavage activity is highly constrained by the NHH rule. In this study, cleavage sites of all target examples were selected such that they would be consistent with the NHH rule to retain HHR cleavage activity. To restrict HHR specificity, our strategy is not feasible when $\mathrm{G}$ exists in one and/or two 5 ' bases upstream of the editing site. The editing sites of all target examples in this study were selected such that they would be consistent with the $\mathrm{NHH}$ rule to retain $\mathrm{HHR}$ cleavage activity (HTR2C: GUA, APOB: AUA, and FLNA: UUC). Another restriction is the combination of the target nucleotide to cleave and not to cleave. For example, A-to-I RNA editing-specific HHRs cannot be redesigned to cleave $A$ and not I by our strategy. Inosine can form a base pair with $\mathrm{U}$ in addition to $\mathrm{A}$ and $\mathrm{C}$, and thus it precludes the use of $U$ as the editing recognition nucleotide. This is also the case for C-to-U-editing recognition, as $\mathrm{G}$ can form a base pair with both $\mathrm{C}$ and $\mathrm{U}$, and thus HHRs would cleave both target RNAs indiscriminately. Despite these limitations, our strategy is applicable to specific cleavage of all other combinations of single substitutions. For the same reason, mutation-specific HHR based on $\mathrm{NHH}$ specificity cannot achieve selective digestion for all variations of nucleotide mutations. However, by choosing a ribozyme design based on our strategy and depending on which base is the target mutation site and the context of its surrounding sequences, nearly any nucleotide substitution can be specifically recognized by the artificial ribozyme. This expansion of specificity increases the application of HHR for mutations or substitutional editing for selective inhibition of gene expression.
We demonstrated that editing-specific cleavage by the ribozyme is applicable for not only short RNA fragments, but also for physiological mRNA in vitro. The efficiency of editing-specific cleavage of cell-derived target mRNA was assessed as a change in the editing ratio by utilizing the peak height of the editing site based on DNA sequencing chromatograms; editing-specific cleavage of HR-FLNA-edit could be observed using this method. Assuming that no background cleavage of the nonedited mRNA occurs, the fractions of editing-specific cleavage can be estimated from the reduction of the editing ratio. Although quantification of the total amount of FLNA mRNA is necessary for a detailed analysis, we estimate that $\sim 51 \%$ of edited-FLNA mRNA was cleaved by HR-FLNA-edit in a 24 -h reaction. The amount of editing-specific cleavage was dependent on the reaction time, but cleavage efficiency was low compared with the reaction against FLNA mRNA fragment, for which the 1-h cleavage reaction was nearly saturated against the FLNA RNA fragment (Fig. 5; Supplemental Fig. S2). Because minimal hammerhead structure is utilized as a basic framework for our ribozymes, our results were consistent with those of previous studies demonstrating that in vitro transcleavage constants in the design of minimal HHR against long substrates were $\sim 100$-fold lower than those with short RNA fragments (Hormes and Sczakiel 2002). The main reason for this decrease in cleavage activity of minimal hammerheads may be the nonspecific interactions with ribozyme nucleotides (Campbell et al. 1997) or the restriction of proper base-pairing when long substrates form higher-order structures (Hormes and Sczakiel 2002).

Although designed minimal HHRs successfully cleave target mRNA in vivo (Goodchild 2002; Citti and Rainaldi 2005; Tedeschi et al. 2009), many studies have shown that ribozyme activity is highly restricted by not only target accessibility but also by the low physiological concentration of $\mathrm{Mg}^{2+}$ (Castanotto et al. 2002). Cleavage activity of the ribozymes in this study significantly decreased at a physiological $\mathrm{Mg}^{2+}$ concentration of $0.1 \mathrm{mM}$ (data not shown). In contrast to the minimal HHR used as a basic framework in our design, most natural HHRs contain a tertiary stabilizing motif formed by the interaction between helices I and II in their short loops (see Fig. 1). Recently, several studies have shown that these tertiary stabilizing motifs increase the cleavage activity against long and structured RNAs and at physiological $\mathrm{Mg}^{+2}$ concentrations (De la Peña et al. 2003; Khvorova et al. 2003; Carbonell et al. 2011). If the recognition base regulates the active formation of ribozyme for recognition of the target-editing site, these tertiary stabilizing motifs may negatively affect our design. However, a basic framework including a tertiary stabilizing motif may be available as an alternative method for developing an editingspecific ribozyme with more effective intracellular applications.

In summary, we developed a ribozyme design for target RNA cleavage depending on A-to-I and C-to-U substitutional RNA editing based on the framework of the minimal 
HHR. These ribozymes altered the recognition base and showed highly specific cleavage activity against both edited synthetic RNA fragments and physiological mRNA in vitro. Our strategy can be applied for selective cleavage in not only substitutional RNA editing but also in analyzing other types of mutations by choosing a specific combination of a recognition base and target base. Our data indicate that our ribozyme design strategy is effective for cleaving target RNA in an editing-dependent manner and provides an experimental basis for specific RNA-editing-dependent target RNA degradation in vivo.

\section{MATERIALS AND METHODS}

\section{General}

Reagents and solvents were purchased from standard suppliers and used without further purification. DNA oligonucleotides were purchased from Genenet Co., Ltd. Substrate RNAs with inosine modifications were purchased from Hokkaido System Science Co., Ltd. Sequences of all purchased DNA oligonucleotides and synthetic RNAs are listed in Supplemental Table S1.

\section{Preparation of ribozymes and substrate RNAs}

All ribozymes were synthesized using an in vitro transcription method with an AmpliScribe T7 Kit (Epicentre Biotechnologies). For the reaction, $1 \mu \mathrm{M}$ of forward DNA oligonucleotide containing the T7 promoter sequence and $1 \mu \mathrm{M}$ of a reverse DNA oligonucleotide were mixed in and annealing buffer (50 mM Tris$\mathrm{HCl}$ at $\mathrm{pH} 7.6$ and $50 \mathrm{mM} \mathrm{NaCl}$ ), and the annealing reaction was performed by denaturing the mixture at $95^{\circ} \mathrm{C}$ for $3 \mathrm{~min}$, followed by cooling to room temperature for $15 \mathrm{~min}$. Next, the annealing product was elongated using Klenow polymerase (New England Biolabs) to generate a double-stranded DNA template. A total of $1 \mu \mathrm{g}$ of template DNA was used for the $20-\mu \mathrm{L}$ transcription reaction. The in vitro transcription reaction was performed according to the manufacturer's protocol. After transcription, the reaction solution was subjected to denaturing gel electrophoresis in $8 \mathrm{M}$ Urea containing $8 \%$ polyacrylamide gel for purification. The corresponding band was excised and the transcribed ribozyme was eluted from the gel by using the crush-and-soak method in TE buffer $(10 \mathrm{mM}$ Tris- $\mathrm{HCl}$ at $\mathrm{pH} 7.5$ and $1 \mathrm{mM}$ ethylenediaminetetraacetic acid [EDTA]). After phenol/chloroform extraction and ethanol precipitation, purified ribozymes were dissolved in TE buffer and quantified by measuring the absorbance at $260 \mathrm{~nm}$ with each molecular coefficient calculated using OligoAnalyzer 3.1 (Integrated DNA Technologies, Inc.). Only substrate RNAs for $H T R 2 C$-ade were synthesized using the oligonucleotide HTR2Cade-F and $H T R 2 C$-ade-R by in vitro transcription according to the same procedure used for ribozyme preparation. Other substrate RNAs were purchased from Hokkaido System Science Co., Ltd.; these sequences are summarized in Supplemental Table S1.

\section{Cleavage reaction and kinetic analysis}

To analyze the cleavage activity of the ribozyme by using gel electrophoresis, substrate RNAs were $5^{\prime}$-end-labeled with $\left[\gamma^{32}\right.$ P]ATP (GE Healthcare) using T4 polynucleotide kinase (New
England Biolabs). Radioactive substrate RNAs were purified using polyacrylamide gel electrophoresis (PAGE) in $8 \mathrm{M}$ urea with a $15 \%$ polyacrylamide gel according to the crush-and-soak method. Cleavage reactions were carried out in cleavage buffer $(20 \mathrm{mM}$ Tris- $\mathrm{HCl}$ at $\mathrm{pH} 7.6,100 \mathrm{mM} \mathrm{NaCl}$, and $20 \mathrm{mM} \mathrm{MgCl}_{2}$ ) at $37^{\circ} \mathrm{C}$ under single-turnover conditions. Excess amounts of ribozyme $(1 \mu \mathrm{M})$ and substrate $(<0.1 \mu \mathrm{M})$ were first mixed in cleavage buffer without $\mathrm{MgCl}_{2}$, and then the annealing reaction was performed by heating at $80^{\circ} \mathrm{C}$ for $3 \mathrm{~min}$ and slowly cooling to $25^{\circ} \mathrm{C}$ at a rate of $1^{\circ} \mathrm{C} / 10 \mathrm{sec}$, followed by incubation at this temperature for $5 \mathrm{~min}$. After taking a zero-time fraction, the cleavage reaction was started by the addition of $20 \mathrm{mM} \mathrm{MgCl}_{2}$. Aliquots were taken at different time points and quenched on ice after adding the same volume of stop solution (10 mM EDTA and 80\% formamide). Substrate and cleavage products were separated by PAGE on $15 \%$ denaturing gels, and the amount of radioactivity in each band was quantified using a Bio-Imaging Analyzer FLA-7000 (Fujifilm). Each cleavage reaction was carried out at least twice to ensure reproducibility. The fraction of cleavage product, $F_{\mathrm{t}}$, at different time points was determined on the basis of corresponding scan data. Pseudofirst-order rate constants of cleavage ( $k$ or $k_{\mathrm{a}}, k_{\mathrm{b}}$ ) were calculated using nonlinear regression analysis with the software Igor Pro (WaveMetrics, Inc.). Data were fitted to the single-exponential equation $F_{\mathrm{t}}=F_{0}+F_{1}\left(1-\mathrm{e}^{-k \mathrm{t}}\right)$, where $F_{0}$ and $F_{1}$ are the product fractions at zero time and at the reaction end point, respectively, and $k$ is the first-order rate constant of cleavage $\left(k_{\text {cat }}\right)$. For data which could not be adequately fitted to a single exponential, the double-exponential equation $F_{\mathrm{t}}=F_{\mathrm{a}}\left(1-\mathrm{e}_{\mathrm{a}}^{-k \mathrm{t}}\right)+F_{\mathrm{b}}\left(1-\mathrm{e}_{\mathrm{b}}^{-k}\right)$ was used for data fitting, where the product fraction and the rate constant for a rapid process are denoted as $F_{\mathrm{a}}$ and $k_{\mathrm{a}}$, respectively, and the product fraction and the rate constant for a slow process are $F_{\mathrm{b}}$ and $k_{\mathrm{b}}$, respectively.

\section{Preparation of edited FLNA mRNA from the cells}

Tet-ADAR2 cells, which are HEK293 cells with a stably transfected ADAR2-expressing sequence under the control of the Tet-on system, were constructed in our laboratory. Tet-ADAR2 cells were cultured as monolayers in Dulbecco's modified Eagle medium (Sigma) supplemented with $10 \%$ Tet system-approved fetal bovine serum (Clontech), $1 \mu \mathrm{g} / \mathrm{mL}$ of puromycin, and $100 \mu \mathrm{g} / \mathrm{mL}$ of G418 (Sigma) at $37^{\circ} \mathrm{C}$ and $5 \% \mathrm{CO}_{2}$. To induce ADAR2 expression, $5 \mu \mathrm{g} /$ $\mathrm{mL}$ of doxycycline (Dox) was added to subconfluent cells $(\sim 80 \%$ of confluence). Next, $96 \mathrm{~h}$ after the addition of Dox, total RNA was extracted by using Sepasol RNA I Super G (Nacalai Tesque) according to the manufacturer's protocol.

\section{Analysis of editing-specific cleavage against cell-extracted FLNA mRNA by using direct sequencing}

The cleavage efficiency of HR-FLNA-edit against FLNA mRNA extracted from Tet-ADAR2 cells was analyzed using the following method. Total RNA (750 ng) extracted from Tet-ADAR2 cells cultured with Dox $(5 \mu \mathrm{g} / \mathrm{mL})$ was annealed in the presence of excess HR-FLNA-edit (final concentration, $2.5 \mu \mathrm{M}$ ) in an annealing buffer ( $50 \mathrm{mM}$ Tris- $\mathrm{HCl}$ at $\mathrm{pH} 7.6,50 \mathrm{mM} \mathrm{NaCl}$ ) by heating at $80^{\circ} \mathrm{C}$ for 3 min and then cooling on ice. The control samples were generated by the same annealing reaction without ribozyme. Additionally, a catalytically inactive HHR (HR-FLNA-mut) was synthesized for 
evaluating the effect on FLNA mRNA stability. HR-FLNA-mut, in which the highly conserved catalytic core sequence CUGA was substituted by CUAA (substituting nucleotide is underlined), was synthesized with the oligonucleotide FLNA-mut_R (Supplemental Table S1) by an in vitro transcription method according to the same procedure used for ribozyme preparation and annealed with the same condition for HR-FLNA-edit. The cleavage reaction was initiated by the addition of $20 \mathrm{mM} \mathrm{MgCl}_{2}$ to the annealing sample, which was incubated for $1,3,6$, and $24 \mathrm{~h}$ at $37^{\circ} \mathrm{C}$. After the cleavage reaction, the RNA samples were treated with $10 \mathrm{U}$ of DNaseI (Takara) for $3 \mathrm{~h}$ at $37^{\circ} \mathrm{C}$, followed by phenol/chloroform extraction and ethanol precipitation. To synthesize FLNA cDNA, $0.5 \mu \mathrm{g}$ of purified RNA was subjected to reverse transcription using the adapter-linked oligo $(\mathrm{dT})_{17}$ primer with the Transcriptor High Fidelity cDNA Synthesis Kit (Roche) according to the manufacturer's protocol. Next, cDNA was amplified using PCR with FLNA-specific primers (FLNAseq_F and FLNAseq_R), and the 310-bp PCR product was purified using $1.5 \%$ agarose gel electrophoresis. To analyze the efficiency of A-to-I RNA editing at the FLNA Q/R site by using a direct sequencing method, the purified PCR product was reacted with BigDye in the Terminator Cycle Sequencing Kit (Applied Biosystems), and then analyzed using a 3130 Genetic Analyzer (Applied Biosystems) to generate sequence chromatograms. The heights of the T and $\mathrm{C}$ peaks at the editing site were measured using the chromatograms generated for the sequences obtained after PCR with the reverse primer FLNAseq_R; the software Sequence Scanner ver. 1.0 (Applied Biosystems) was used for analysis. Each editing ratio was calculated on the basis of the peak height of $\mathrm{C}$ divided by the peak height of $\mathrm{C}+\mathrm{T}$.

\section{SUPPLEMENTAL MATERIAL}

Supplemental material is available for this article.

\section{ACKNOWLEDGMENTS}

This work was supported in part by a Grant-in-Aid for Young Scientists (B) from the Japan Society for the Promotion of Science (No. 23710263) and funds from the Central Research Institute of Fukuoka University (No. 117106).

Received March 25, 2012; accepted June 7, 2012.

\section{REFERENCES}

Bass BL. 2002. RNA editing by adenosine deaminases that act on RNA. Annu Rev Biochem 71: 817-846.

Blanc V, Davidson NO. 2003. C-to-U RNA editing: mechanisms leading to genetic diversity. J Biol Chem 278: 1395-1398.

Burns CM, Chu H, Rueter SM, Hutchinson LK, Canton H, SandersBush E, Emeson RB. 1997. Regulation of serotonin-2C receptor G-protein coupling by RNA editing. Nature 387: 303-308.

Campbell TB, McDonald CK, Hagen M. 1997. The effect of structure in a long target RNA on ribozyme cleavage efficiency. Nucleic Acids Res 25: 4985-4993.

Carbonell A, Flores R, Gago S. 2011. Trans-cleaving hammerhead ribozymes with tertiary stabilizing motifs: in vitro and in vivo activity against a structured viroid RNA. Nucleic Acids Res 39: 2432-2444.

Castanotto D, Li JR, Michienzi A, Langlois MA, Lee NS, Puymirat J, Rossi JJ. 2002. Intracellular ribozyme applications. Biochem Soc Trans 30: 1140-1145.
Chen SH, Habib G, Yang CY, Gu ZW, Lee BR, Weng SA, Silberman SR, Cai SJ, Deslypere JP, Rosseneu M, et al. 1987. Apolipoprotein B-48 is the product of a messenger RNA with an organ-specific inframe stop codon. Science 238: 363-366.

Chester A, Scott J, Anant S, Navaratnam N. 2000. RNA editing: cytidine to uridine conversion in apolipoprotein B mRNA. Biochim Biophys Acta 1494: 1-13.

Citti L, Rainaldi G. 2005. Synthetic hammerhead ribozymes as therapeutic tools to control disease genes. Curr Gene Ther 5: 11-24.

Dawson PA, Marini JC. 2000. Hammerhead ribozymes selectively suppress mutant type I collagen mRNA in osteogenesis imperfecta fibroblasts. Nucleic Acids Res 28: 4013-4020.

De la Peña M, Gago S, Flores R. 2003. Peripheral regions of natural hammerhead ribozymes greatly increase their self-cleavage activity. EMBO J 22: 5561-5570.

Eggington JM, Greene T, Bass BL. 2011. Predicting sites of ADAR editing in double-stranded RNA. Nat Commun 2: 319. doi: $10.1038 /$ ncomms 1324 .

Farajollahi S, Maas S. 2010. Molecular diversity through RNA editing: a balancing act. Trends Genet 26: 221-230.

Gommans WM, Tatalias NE, Sie CP, Dupuis D, Vendetti N, Smith L, Kaushal R, Maas S. 2008. Screening of human SNP database identifies recoding sites of A-to-I RNA editing. RNA 14: 20742085.

Goodchild J. 2002. Hammerhead ribozymes for target validation. Expert Opin Ther Targets 6: 235-247.

Haseloff J, Gerlach WL. 1988. Simple RNA enzymes with new and highly specific endoribonuclease activities. Nature 334: 585-591.

Higuchi M, Single FN, Kohler M, Sommer B, Sprengel R, Seeburg PH. 1993. RNA editing of AMPA receptor subunit GluR-B: a basepaired intron-exon structure determines position and efficiency. Cell 75: 1361-1370.

Hormes R, Sczakiel G. 2002. The size of hammerhead ribozymes is related to cleavage kinetics: the role of substrate length. Biochimie 84: 897-903.

Khvorova A, Lescoute A, Westhof E, Jayasena SD. 2003. Sequence elements outside the hammerhead ribozyme catalytic core enable intracellular activity. Nat Struct Biol 10: 708-712.

Koizumi M, Ohtsuka E. 1991. Effects of phosphorothioate and 2 -amino groups in hammerhead ribozymes on cleavage rates and $\mathrm{Mg}^{2+}$ binding. Biochemistry 30: 5145-5150.

Kore AR, Vaish NK, Kutzke U, Eckstein F. 1998. Sequence specificity of the hammerhead ribozyme revisited; the NHH rule. Nucleic Acids Res 26: 4116-4120.

Levanon EY, Hallegger M, Kinar Y, Shemesh R, Djinovic-Carugo K, Rechavi G, Jantsch MF, Eisenberg E. 2005. Evolutionarily conserved human targets of adenosine to inosine RNA editing. Nucleic Acids Res 33: 1162-1168.

Lewin AS, Drenser KA, Hauswirth WW, Nishikawa S, Yasumura D, Flannery JG, LaVail MM. 1998. Ribozyme rescue of photoreceptor cells in a transgenic rat model of autosomal dominant retinitis pigmentosa. Nat Med 4: 967-971.

Li JB, Levanon EY, Yoon JK, Aach J, Xie B, Leproust E, Zhang K, Gao Y, Church GM. 2009. Genome-wide identification of human RNA editing sites by parallel DNA capturing and sequencing. Science 324: $1210-1213$.

Ludwig J, Blaschke M, Sproat BS. 1998. Extending the cleavage rules for the hammerhead ribozyme: mutating adenosine ${ }^{15.1}$ to inosine ${ }^{15.1}$ changes the cleavage site specificity from $\mathrm{N}^{16.2} \mathrm{U}^{16.1} \mathrm{H}^{17}$ to $\mathrm{N}^{16.2} \mathrm{C}^{16.1} \mathrm{H}^{17}$. Nucleic Acids Res 26: 2279-2285.

Maas S, Rich A. 2000. Changing genetic information through RNA editing. Bioessays 22: 790-802.

Maas S, Kawahara Y, Tamburro KM, Nishikura K. 2006. A-to-I RNA editing and human disease. RNA Biol 3: 1-9.

Nishikura K. 2010. Functions and regulation of RNA editing by ADAR deaminases. Annu Rev Biochem 79: 321-349.

Nishimoto Y, Yamashita T, Hideyama T, Tsuji S, Suzuki N, Kwak S. 2008. Determination of editors at the novel A-to-I editing positions. Neurosci Res 61: 201-206. 


\section{Fukuda et al.}

Nurpeisov V, Hurwitz SJ, Sharma PL. 2003. Fluorescent dye terminator sequencing methods for quantitative determination of replication fitness of human immunodeficiency virus type 1 containing the codon 74 and 184 mutations in reverse transcriptase. J Clin Microbiol 41: 3306-3311.

Perriman R, Delves A, Gerlach WL. 1992. Extended target-site specificity for a hammerhead ribozyme. Gene 113: 157-163.

Powell LM, Wallis SC, Pease RJ, Edwards YH, Knott TJ, Scott J. 1987. A novel form of tissue-specific RNA processing produces apolipoprotein-B48 in intestine. Cell 50: 831-840.

Pullirsch D, Jantsch MF. 2010. Proteome diversification by adenosine to inosine RNA editing. RNA Biol 7: 205-212.

Riedmann EM, Schopoff S, Hartner JC, Jantsch MF. 2008. Specificity of ADAR-mediated RNA editing in newly identified targets. RNA 14: $1110-1118$.

Scherr M, Grez M, Ganser A, Engels JW. 1997. Specific hammerhead ribozyme-mediated cleavage of mutant $\mathrm{N}$-ras mRNA in vitro and ex vivo. Oligoribonucleotides as therapeutic agents. J Biol Chem 272: 14304-14313.

Scott WG, Finch JT, Klug A. 1995. The crystal structure of an all-RNA hammerhead ribozyme: a proposed mechanism for RNA catalytic cleavage. Cell 81: 991-1002.

Seeburg PH, Hartner J. 2003. Regulation of ion channel/neurotransmitter receptor function by RNA editing. Curr Opin Neurobiol 13: 279-283.
Serra MJ, Smolter PE, Westhof E. 2004. Pronounced instability of tandem IU base pairs in RNA. Nucleic Acids Res 32: 1824-1828.

Sommer B, Kohler M, Sprengel R, Seeburg PH. 1991. RNA editing in brain controls a determinant of ion flow in glutamate-gated channels. Cell 67: 11-19.

Tedeschi L, Lande C, Cecchettini A, Citti L. 2009. Hammerhead ribozymes in therapeutic target discovery and validation. Drug Discov Today 14: 776-783.

Uhlenbeck OC. 1987. A small catalytic oligoribonucleotide. Nature 328: 596-600.

Wang AB, Liu DP, Liang CC. 2003. Regulation of human apolipoprotein B gene expression at multiple levels. Exp Cell Res 290: 112.

Werner M, Uhlenbeck OC. 1995. The effect of base mismatches in the substrate recognition helices of hammerhead ribozymes on binding and catalysis. Nucleic Acids Res 23: 2092-2096.

Werry TD, Loiacono R, Sexton PM, Christopoulos A. 2008. RNA editing of the serotonin $5 \mathrm{HT}_{2 \mathrm{C}}$ receptor and its effects on cell signalling, pharmacology and brain function. Pharmacol Ther 119: 7-23.

Zoumadakis M, Tabler M. 1995. Comparative analysis of cleavage rates after systematic permutation of the NUX $\downarrow$ consensus target motif for hammerhead ribozymes. Nucleic Acids Res 23: 1192-1196.

Zoumadakis M, Neubert WJ, Tabler M. 1994. The influence of imperfectly paired helices I and III on the catalytic activity of hammerhead ribozymes. Nucleic Acids Res 22: 5271-5278. 

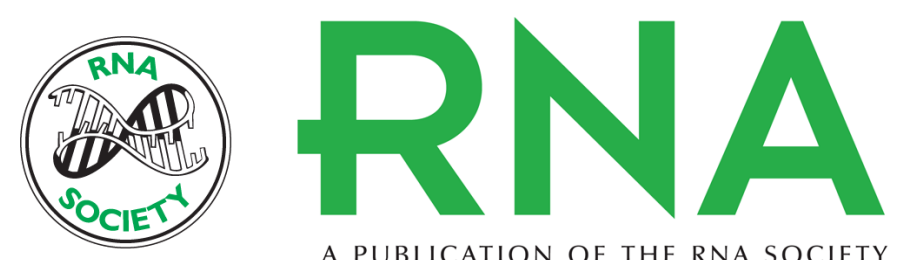

A PUBLICATION OF THE RNA SOCIETY

\section{A strategy for developing a hammerhead ribozyme for selective RNA cleavage depending on substitutional RNA editing}

Masatora Fukuda, Kei Kurihara, Yasuyoshi Tanaka, et al.

RNA 2012 18: 1735-1744 originally published online July 13, 2012

Access the most recent version at doi:10.1261/rna.033399.112

Supplemental
Material http://rnajournal.cshlp.org/content/suppl/2012/06/26/rna.033399.112.DC1

References This article cites 46 articles, 8 of which can be accessed free at:

http://rnajournal.cshlp.org/content/18/9/1735.full.html\#ref-list-1

License

Email Alerting Receive free email alerts when new articles cite this article - sign up in the box at the Service top right corner of the article or click here. 